\title{
Quantitative approaches in production management
}

\author{
Stefan Helber ${ }^{1} \cdot$ Ton de Kok ${ }^{2} \cdot$ Heinrich Kuhn ${ }^{3} \cdot$ Michael Manitz ${ }^{4}$. \\ Andrea Matta ${ }^{5} \cdot$ Raik Stolletz ${ }^{6}$
}

Published online: 25 November 2019

(c) Springer-Verlag GmbH Germany, part of Springer Nature 2019

Production management requires Operations Research models and methods for undertaking tactical decisions such as the optimal dimensioning of capacity during the design phase of production systems as well as operational decisions such as scheduling of operations facing dynamic demands that have to be met. Production companies, largescale industrial ones as well as medium-size and small-lot producers aim at providing goods and services to the customers gain added value over the entire supply chain. The objectives are reducing costs and/or increasing revenues, benefits, and profits by optimizing the production infrastructure and the production processes. The consideration of uncertainties, scarce capacities, and many other constraints make production management a challenging task from the practical and the theoretical perspective.

This Special Issue of OR Spectrum is dedicated to Horst Tempelmeier who, after being in academia for more than 40 years, will retire as a Professor for Production Economics and Management at the University of Cologne. Horst has been active in quantitative production management in general (see Tempelmeier and ReithAhlemeier (2004); Tempelmeier (2002, 1997) for MRP/ERP issues) and in particular in the fields of (multi-level) lot-sizing under capacity restrictions and/or stochastic demand (see Copil et al. 2017; Tempelmeier and Copil 2016; Hilger et al. 2016; Tempelmeier and Hilger 2015; Tempelmeier 2013b, 2011a; Tempelmeier and Herpers 2011, 2010; Sahling et al. 2009; Tempelmeier and Buschkühl 2009, 2008; Tempelmeier and Derstroff 1996; Tempelmeier and Helber 1994) as well as the design of flow lines using queueing-model-based performance analysis (see Lagershausen et al. 2013; Manitz and Tempelmeier 2012; Tempelmeier 2003; Tempelmeier and Bürger 2001; Tempelmeier and Kuhn 1992; Tempelmeier et al. 1989). Furthermore, Horst is the author of a number of textbooks such as Tempelmeier and Kuhn (1993) and Tempelmeier (2011b), which significantly contributed to the teaching and the practice of quantitative production management.

We are honored to serve as guest editors of the special issue on quantitative approaches in production management. For four of us, Horst has been the or a main academic advisor. Andrea represents Horst's link to the SMMSO research commu-

Michael Manitz

michael.manitz@uni-duisburg-essen.de

Extended author information available on the last page of the article 
nity on stochastic models for manufacturing and service operations (Liberopoulos et al. 2010), and Ton stands for Horst's research on inventory control and dynamic lot-sizing (see Tempelmeier and Fischer 2018; Tempelmeier and Bantel 2015; Tempelmeier 2013a; Tempelmeier and Fischer 2010; Tempelmeier 2007, 2006, 2000, 1993, 1985). We thank Horst for fruitful and controversial academic discussions, for his guidance, and for his inspiration.

For this special issue, more than 30 papers have been submitted of which the following six papers have been accepted: Benda et al. (2019) present a machine-learning approach for minimizing the makespan in an hybrid flow shop with parallel stations, sequence-dependent setup times, and limited transport resources, and, hence, possible blocking. Briskorn and Zeise (2019) propose a cyclic production scheme for a make and pack production process under uncertain demand and capacitated storage. Hottenrott and Grunow (2019) show how flexible layouts of assembly lines instead of strong serially arranged ones may improve the efficiency under increasing product heterogeneity, especially in the automotive industry. Kloos et al. (2019) analyze optimization strategies for the allocation of scarce supply among different sales hierarchies under service-level constraints. Stadtler and Meistering (2019) present deterministic lot-sizing models with different service-level constraints whereby some measure turns out to be more or less appropriate. Tan (2019) reveals the optimality of a hedging policy in a make-to-stock environment via a Markov-chain model with continuous material flow.

We thank the authors and the reviewers for contributing to this special issue which we hope will foster the use of quantitative approaches in production management.

\section{References}

Benda F, Braune R, Dörner KF, Hartl RF (2019) A machine learning approach for flow shop scheduling problems with alternative resources, sequence-dependent setup times, and blocking. OR Spectr 41(4) (to appear in this issue)

Briskorn D, Zeise P (2019) A cyclic production scheme for the synchronized and integrated two-level lotsizing and scheduling problem with no-wait restrictions and stochastic demand. OR Spectr. https:// doi.org/10.1007/s00291-019-00555-y

Copil K, Wörbelauer M, Meyr H, Tempelmeier H (2017) Simultaneous lotsizing and scheduling problems: a classification and review of models. OR Spectr 39(1):1-64

Hilger T, Sahling F, Tempelmeier H (2016) Capacitated dynamic production and remanufacturing planning under demand and return uncertainty. OR Spectr 38(4):849-876

Hottenrott A, Grunow M (2019) Flexible layouts for the mixed-model assembly of heterogeneous vehicles. OR Spectr. https://doi.org/10.1007/s00291-019-00556-X

Kloos K, Pibernik R, Schulte B (2019) Allocation planning in sales hierarchies with stochastic demand and service-level targets. OR Spectr 41(4) (to appear in this issue, published online 2018-12-28)

Lagershausen S, Manitz M, Tempelmeier H (2013) Performance analysis of closed-loop automotive assembly lines with general processing times and finite buffer spaces. IIE Trans 45(5):502-515

Liberopoulos G, Papadopoulos C, Smith JM, Tempelmeier H, Tolio T (2010) New developments in stochastic models of manufacturing and service operations. Int J Prod Res 54(20):1-3

Manitz M, Tempelmeier H (2012) The variance of inter-departure times of the output of an assembly line with finite buffers, converging flow of material, and general service times. OR Spectr 34(1):273-291

Sahling F, Buschkühl L, Tempelmeier H, Helber S (2009) Solving a multilevel capacitated lot sizing problem with multi-period setup carry-over via a fix-and-optimize heuristic. Comput Oper Res 36(9):25462553 
Stadtler H, Meistering M (2019) Model formulations for the capacitated lot-sizing problem with servicelevel constraints. OR Spectr. https://doi.org/10.1007/s00291-019-00552-1

Tan B (2019) Production control with price, cost, and demand uncertainty. OR Spectr 41(4) (to appear in this issue, published online 2018-12-28)

Tempelmeier H (1985) Inventory control using a service constraint on the expected customer order waiting time. Eur J Oper Res 19(3):313-323

Tempelmeier H (1993) Safety stock allocation in a two-echelon distribution system. Eur J Oper Res 65:96117

Tempelmeier H (1997) Resource-constrained materials requirements planning-MRP ${ }^{\mathrm{rc}}$. Prod Plan Control $8(5): 451-461$

Tempelmeier H (2000) Inventory service-levels in the customer supply chain. OR Spectr 22(3):361-380

Tempelmeier H (2002) A simple heuristic for dynamic order sizing and supplier selection with time-varying data. Prod Oper Manag 11(4):499-515

Tempelmeier H (2003) Practical considerations in the optimization of flow production systems. Int J Prod Res 41(1): 149-170

Tempelmeier H (2006) Supply chain inventory optimization with two customer classes. Eur J Oper Res 174(1):600-621

Tempelmeier H (2007) On the stochastic uncapacitated dynamic single-item lotsizing problem with service level constraints. Eur J Oper Res 181(1):184-194

Tempelmeier H (2011a) A column generation heuristic for dynamic capacitated lot sizing with random demand under a fill rate constraint. Omega 39(6):627-633

Tempelmeier H (2011b) Inventory management in supply networks-problems, models, solutions, 2nd edn. Books on Demand, Norderstedt

Tempelmeier H (2013a) A multi-level inventory system with a make-to-order supplier. Int J Prod Res 51:6880-6890

Tempelmeier H (2013b) Stochastic lot sizing problems. In: Smith JM, Tan B (eds) Handbook of stochastic models and analysis of manufacturing system operations. Springer, Berlin, pp 113-143

Tempelmeier H, Bantel O (2015) Integrated optimization of safety stock and transportation capacity. Eur J Oper Res 247(1):101-112

Tempelmeier H, Bürger M (2001) Performance evaluation of unbalanced flow lines with general distributed processing times, failures and imperfect production. IIE Trans 33(4):293-302

Tempelmeier H, Buschkühl L (2008) Dynamic multi-machine lotsizing and sequencing with simultaneous scheduling of a common setup resource. Int J Prod Econ 113(1):401-412

Tempelmeier H, Buschkühl L (2009) A heuristic for the dynamic multi-level capacitated lotsizing problem with linked lotsizes for general product structures. OR Spectr 31(2):385-404

Tempelmeier H, Copil K (2016) Capacitated lot sizing with parallel machines, sequence-dependent setups, and a common setup operator. OR Spectr 38(4):810-847

Tempelmeier H, Derstroff M (1996) A lagrangean-based heuristic for dynamic multilevel multi-item constrained lot sizing. Manag Sci 42(5):738-757

Tempelmeier H, Fischer L (2010) Approximation of the probability distribution of the customer waiting time under an $(r, s, q)$ inventory policy in discrete time. Int J Prod Res 48(21):6275-6291

Tempelmeier H, Fischer L (2018) A procedure for the approximation of the waiting time distribution in a discrete-time $(r, S)$ inventory system. Int J Prod Res 57(5):1413-1426

Tempelmeier H, Helber S (1994) A heuristic for dynamic multi-item multi-level capacitated lotsizing for general product structures. Eur J Oper Res 75(2):296-311

Tempelmeier $\mathrm{H}$, Herpers $\mathrm{S}$ (2010) $\mathrm{ABC}_{\beta}$-a heuristic for dynamic capacitated lot sizing with random demand under a fillrate constraint. Int J Prod Res 48(17):5181-5193

Tempelmeier H, Herpers S (2011) Dynamic uncapacitated lot sizing with random demand under a fillrate constraint. Eur J Oper Res 212(3):497-507

Tempelmeier H, Hilger T (2015) Linear programming models for a stochastic dynamic capacitated lot sizing problem. Comput Oper Res 59:119-125

Tempelmeier H, Kuhn H (1992) OR-Modelle zur Planung flexibler Fertigungssysteme-Ein Überblick. OR Spectr 14(4):177-192

Tempelmeier H, Kuhn H (1993) Flexible manufacturing systems - decision support for design and operation. Wiley, New York

Tempelmeier H, Reith-Ahlemeier G (2004) Supply chain management and reverse logistics. In: Lackes R, Reese J, Dyckhoff H (eds) Advanced purchasing and order optimization. Springer, Berlin, pp 349-370 
Tempelmeier H, Kuhn H, Tetzlaff U (1989) Performance evaluation of flexible manufacturing systems with blocking. Int J Prod Res 27(11):1963-1979

Publisher's Note Springer Nature remains neutral with regard to jurisdictional claims in published maps and institutional affiliations.

\section{Affiliations}

\section{Stefan Helber ${ }^{1}$ - Ton de Kok ${ }^{2}$. Heinrich Kuhn ${ }^{3}$ - Michael Manitz ${ }^{4}$. Andrea Matta ${ }^{5}$. Raik Stolletz ${ }^{6}$}

Stefan Helber

stefan.helber@prod.uni-hannover.de

Ton de Kok

a.g.d.kok@tue.nl

Heinrich Kuhn

heinrich.kuhn@ku-eichstaett.de

Andrea Matta

andrea.matta@polimi.it

Raik Stolletz

stolletz@bwl.uni-mannheim.de

1 Institute of Production Management, Faculty of Economics and Management, Leibniz University Hannover, Königsworther Platz 1, 30167 Hannover, Germany

2 Department of Operations Planning Accounting and Control, Faculty of Industrial Engineering and Innovation Sciences, Eindhoven University of Technology, Atlas 4.401, $5600 \mathrm{MB}$

Eindhoven, The Netherlands

3 Department of Business Administration, Economics Faculty Ingolstadt, Catholic University of Eichstätt-Ingolstadt, Auf der Schanz 49, 85049 Ingolstadt, Germany

4 Department of Production and Supply Chain Management, Faculty of Business Economics, Mercator School of Management, University of Duisburg/Essen, Lotharstr. 65, 47057 Duisburg, Germany

5 Department of Mechanical Engineering, Politecnico di Milano, Campus Bovisa Sud, via La Masa 1, 20156 Milan, Italy

6 Chair of Production Management, Business School, University of Mannheim, Schloss 68131, Mannheim, Germany 